\title{
Yoga on our minds: a systematic review of yoga for neuropsychiatric disorders
}

\author{
Meera Balasubramaniam $^{1}{ }^{*}$, Shirley Telles $^{2}$ and P. Murali Doraiswamy ${ }^{1,3}$ * \\ ${ }^{1}$ Department of Psychiatry and Behavioral Sciences, Duke University School of Medicine, Durham, NC, USA \\ 2 Indian Council of Medical Research Center for Advanced Research in Yoga and Patanjali Research Foundation, Bengaluru, India \\ ${ }^{3}$ Duke Institute for Brain Sciences, Durham, NC, USA
}

\section{Edited by:}

Susan A. Everson-Rose, University of Minnesota, USA

\section{Reviewed by:}

Dusan Kolar, Queen's University,

Canada

Felicia Iftene, Queens University,

Canada

*Correspondence:

Meera Balasubramaniam

Department of Psychiatry and

Behavioral Sciences, Duke University

School of Medicine, Durham, NC,

USA.

e-mail:meera.balasubramaniam@

duke.edu;

P. Murali Doraiswamy, DUMC-3018,

Department of Psychiatry, Duke

University Medical Center, Durham,

NC 27710, USA.

e-mail:dorai001@mc.duke.edu
Background: The demand for clinically efficacious, safe, patient acceptable, and costeffective forms of treatment for mental illness is growing. Several studies have demonstrated benefit from yoga in specific psychiatric symptoms and a general sense of wellbeing.

Objective: To systematically examine the evidence for efficacy of yoga in the treatment of selected major psychiatric disorders.

Methods: Electronic searches of The Cochrane Central Register of Controlled Trials and the standard bibliographic databases, MEDLINE, EMBASE, and PsycINFO, were performed through April 2011 and an updated in June 2011 using the keywords yoga AND psychiatry OR depression OR anxiety OR schizophrenia OR cognition OR memory OR attention AND randomized controlled trial (RCT). Studies with yoga as the independent variable and one of the above mentioned terms as the dependent variable were included and exclusion criteria were applied.

Results: The search yielded a total of 124 trials, of which 16 met rigorous criteria for the final review. Grade B evidence supporting a potential acute benefit for yoga exists in depression (four RCTs), as an adjunct to pharmacotherapy in schizophrenia (three RCTs), in children with ADHD (two RCTs), and Grade C evidence in sleep complaints (three RCTs). RCTs in cognitive disorders and eating disorders yielded conflicting results. No studies looked at primary prevention, relapse prevention, or comparative effectiveness versus pharmacotherapy.

Conclusion: There is emerging evidence from randomized trials to support popular beliefs about yoga for depression, sleep disorders, and as an augmentation therapy. Limitations of literature include inability to do double-blind studies, multiplicity of comparisons within small studies, and lack of replication. Biomarker and neuroimaging studies, those comparing yoga with standard pharmaco- and psychotherapies, and studies of long-term efficacy are needed to fully translate the promise of yoga for enhancing mental health.

Keywords: yoga, meditation, depression, schizophrenia, cognition, ADHD, clinical trials, alternative medicine

\section{BACKGROUND}

Mental illnesses are asignificant global health concern, despite improvements in treatment modalities and access to care. The World Health Organization (WHO, 2011) has estimated that psychiatric disorders are the leading costs of disability adjusted life years world-wide, with recent figures indicating that $37 \%$ of the loss of healthy years from non-communicable diseases is from mental illnesses. The National Co-morbidity survey replication conducted in the United States estimated the 1-year prevalence of any psychiatric disorder to be $26.2 \%$ (Kessler et al., 2008). According to the WHO, depression ranked third among global disease burdens all over the world in 2004; it was reportedly the most important cause in middle and high income countries, while it ranked eight among the low income countries (World
Health Organization, 2008). Depression was found to result in the greatest decrement in health, compared to asthma, angina, arthritis, and diabetes (Maussavi et al., 2007). Prevalence data for anxiety disorders, suggests that the lifetime prevalence and 12 month prevalence for any anxiety disorder are over 15 and $10 \%$, respectively, with higher prevalence in developed countries (Kessler et al., 2009). Likewise, schizophrenia has been associated with significantly higher health care costs, unemployment rate, and morbidity (Goeree et al., 2005). Sleep complaints are often associated with a variety of psychiatric disorders. About $9-21 \%$ of the population has been estimated to have insomnia accompanied by serious day-time consequences which include chronic fatigue, irritability, low mood, memory impairments, and interpersonal difficulties (Moul et al., 2002). This problem has reached epidemic 
proportions in the United States, where almost $25 \%$ of adults consume sleep medications at some point in a year (National Sleep Foundation, 2005).

The availability of psychopharmacological treatments has increased, but the response and tolerability remain unpredictable and inconsistent. While psychotropics agents can be lifesaving for many people, there remains a considerable unmet need. The landmark National Institute of Mental health (NIMH) funded Sequenced Treatment Alternatives to Relieve Depression $(\mathrm{STAR} * \mathrm{D})$ study showed remission in only one third of major depression patients after a trial with the first anti-depressant and worsening response rates with each subsequent trial (Trivedi et al., 2006). The Primary Care study conducted by WHO found that $60 \%$ of the patients continued to meet criteria for depression after a year of being treated with an anti-depressant (Goldberg et al., 1998). The Clinical Anti-psychotic Trials of Intervention Effectiveness (CATIE) demonstrated that $74 \%$ of the participants discontinued from their treatments in 18 months, with a mean time to discontinuation of 4.6 months (Lieberman et al., 2005). Treatment resistance is a growing problem and there are millions of patients world-wide whose depression, anxiety, or schizophrenia is not fully resolved despite multiple trials of psychopharmacologic agents. Psychotropic medications are costly and suffer from significant side effects leaving patients and clinicians to struggle to balance efficacy against cost and side effects, which often leads to poor compliance and relapse.

Given the heterogeneous nature of psychiatric conditions, with respect to biological, psychological, and social factors, it is not surprising that available standard treatments often have inconsistent response rates. The quest and demand for non-pharmacological treatment modalities has been increasing (Barrows and Jacobs, 2002). A study conducted by the Harris Interactive Service Bureau revealed that 15.8 million adults in the United States practice yoga, triple the number in 2004. The holistic goal of yoga to promote physical and mental health, and also be spiritually and socially conscious, may appeal both to consumers and providers who are concerned about the symptom reduction based focus of psychopharmacology and finding inner peace (Uebelacker et al., 2010). The barriers to access are low and the diversity of practice styles and settings (e.g., at home versus in gyms versus outdoors) allows considerable degree of personalization. Hence, yoga appears to be a well suited intervention to test as a potential therapy for major psychiatric disorders. However, yoga has also become such a cultural phenomenon that it has become difficult for physicians and consumers to differentiate legitimate claims from hype. Our goal in this review was to examine whether the evidence matched the promise.

Yoga, with origins in ancient India has several sub-types (Table 1; Cook, n.d.), and incorporates physical postures (asanas), controlled breathing (pranayama), deep relaxation, and meditation (Javnbakht et al., 2009). In addition to low barriers to access, the scientific rationale for yoga effects on the mind are quite strong. All yoga practices are known to influence the mental state (Telles, 2010) - studies have noted benefits in children (Manjunath and Telles, 2004), adults (Vialatte et al., 2008), elderly (Krishnamurthy and Telles, 2007), and individuals with occupational stress (Vempati and Telles, 2000). In healthy individuals, biomarker studies suggest that yoga influences neurotransmitters, inflammation, oxidative stress, lipids, growth factors, and second messengers (Figure 1), in a manner largely similar to what has been shown for anti-depressants and psychotherapy. It is hypothesized that yoga combines the effects of physical postures, which have been independently associated with mood changes (Phillips et al., 2003), and meditation which increases the levels of Brain-derived neurotrophic factor (BDNF; Xiong and Doraiswamy, 2009). Other effects that have been noted include increased vagal tone, increased gamma-aminobutyric acid (GABA) levels, increase in serum prolactin, downregulation of the hypothalamic-pituitary-adrenal axis and decrease in serum cortisol, and promotion of frontal electroencephalogram (EEG) alpha wave activity which improves relaxation (Janakiramaiah et al., 1998, 2000; Kamei et al., 2000; Streeter et al., 2007). Lastly, prior clinical studies have noted several psychiatric conditions for which yoga has proved beneficial (Shannahoff-Khalsa et al., 1999; Carei et al., 2010; Visceglia and Lewis, 2011; Katzman et al., 2012; Libby et al., 2012) but because of differing methods there is a need to try to synthesize such data to further the field.

Thus, while the effects of yoga on the spiritual aspects of the mind (e.g., inner peace) are well documented, its effects in major clinical psychiatric disorders are less so. The objective of this report was to systematically review the available literature for the effects of yoga on major psychiatric disorders. The focus of this review was primarily categorical disease threshold outcomes (e.g., major depression), in keeping with how psychiatric disorders are categorized and treated, and how effects of psychopharmacologic interventions are assessed - rather than on single symptom domains such as mood or sleep which cut across multiple diagnoses. We did use symptoms (e.g., depression and memory) as search terms to ensure our search was comprehensive but restricted our final review to major disorders that require intervention in practice.

\section{METHODS \\ SEARCH STRATEGY}

Electronic searches of The Cochrane Central Register of Controlled Trials (CENTRAL) and the standard bibliographic databases, MEDLINE, EMBASE, and PsycINFO, was conducted through April 2011 and updated in June 2011, using the keywords yoga AND psychiatry OR depression OR anxiety OR schizophrenia OR cognition OR memory OR attention AND randomized controlled trial (RCT). The title and abstract of each citation were screened based on definite pre-specified inclusion and exclusion criteria. Full text reading of articles that were potentially eligible was undertaken. When full-texts were not available, attempts were made to contact the author. If a reply was not received within 2 weeks from the corresponding author, abstracts were read to check if they had the required information. Studies have been reviewed by all authors and disagreements were resolved by consensus.

Randomized clinical trials with any sub-type of yoga as the intervention and one or more of the above mentioned conditions as the outcome of interest were included. Open trials, nonrandomized trials, case series, and dissertations were excluded. The review includes studies in which subjects have either been formally diagnosed with a disorder or have reported symptoms suggestive of the same. Since age is an important risk factor for cognitive 
Table 1 | Table showing the key elements of the different forms of yoga (Cook, n.d.).

\section{Type of yoga}

\section{Ashtanga yoga}

Hatha yoga

lyengar yoga

Power yoga

Jivamukti yoga

Kali Ray TriYoga

White Lotus Yoga

Integrated yoga therapy

Viniyoga

\section{Svaroopa}

Bikram Yoga (Hot Yoga)

Phoenix rising yoga therapy

Sivananda yoga

Integral yoga

Ananda yoga

Kundalini yoga

ISHTA yoga

Kripalu yoga

Anusara yoga

Tibetan yoga

\section{Key features}

Fast-paced series of sequential posture, based on six series of asanas

Basic form of yoga which incorporates postures, regulated breathing, and meditation

Focuses on the precise alignment of postures

Westernization of Ashtanga yoga. Popular in the US

Physically challenging postures, highly meditative

Consists of flowing, dance-like movements, often accompanied by music

Consists of flowing movements with varying difficulty levels

Designed for medical problems. May include meditation and guided imagery

Gentle practice which particularly emphasizes on the synchronization of poses with breathing

Emphasizes on the "opening of the spine beginning at the tailbone progressing through each spinal area"

Consists of a series of 26 postures performed in a space with temperature above $100^{\circ} \mathrm{F}$

Combines traditional yoga with client centered and mind-body psychology, that incorporates non-directive dialog

Consists of 12 basic yoga postures along with chanting and meditation

Consists of basic hatha yoga postures

Consists of basic hatha yoga postures with use of "silent affirmations while holding up a pose"

Focuses on awakening the energy at the base of the spine and channeling it upwards

Combination of Ashtanga and lyengar yoga

Consists of three stages namely willful practice, willful surrender, and meditation in motion

Consists of basic hatha yoga postures but emphasizes on attitude, alignment, and action

Composed of fine, flowing movements, and controlled breathing
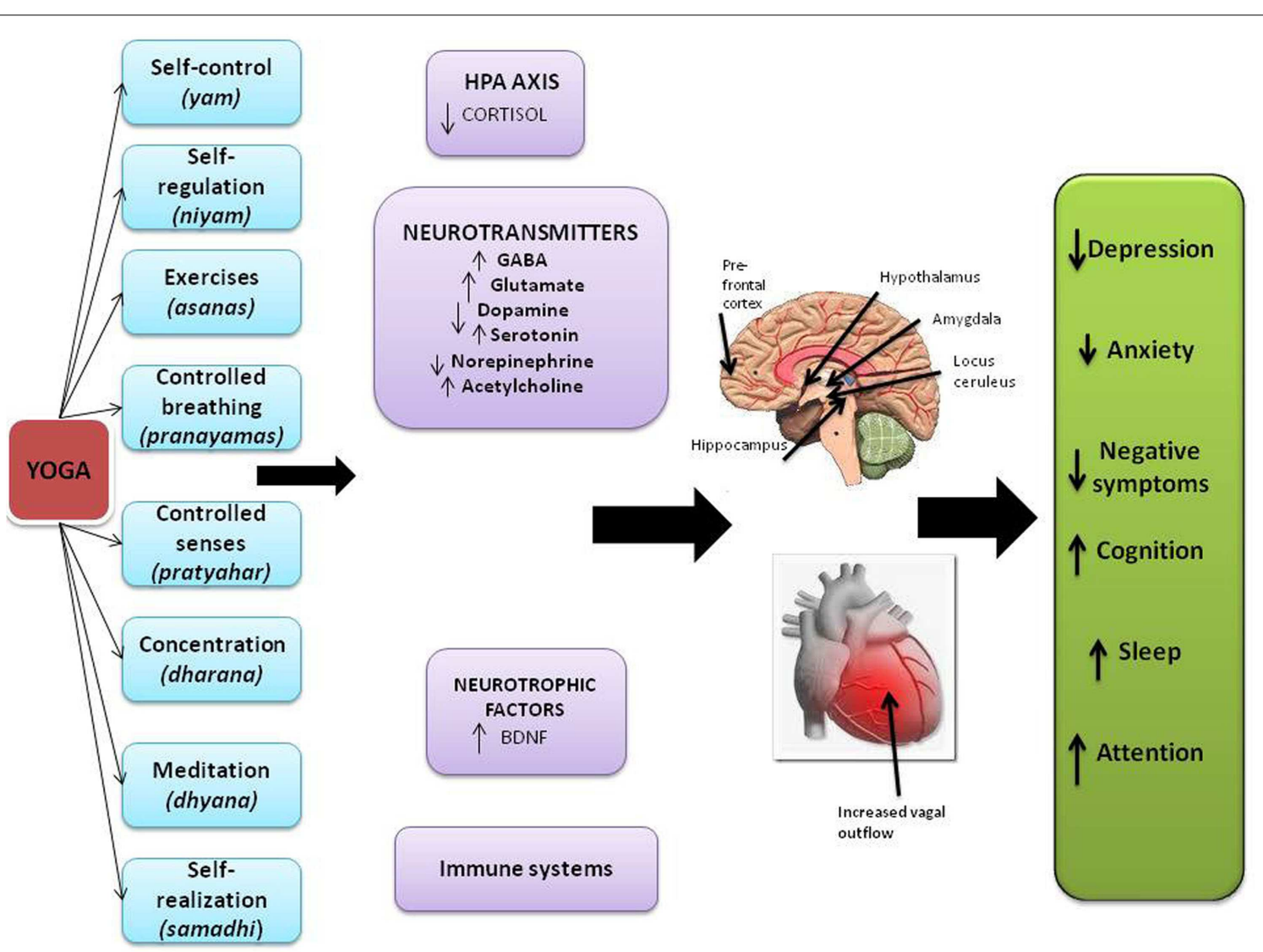

outflow

Immune systems

NEUROTROPHIC

FACTORS

$\uparrow$ BDNF
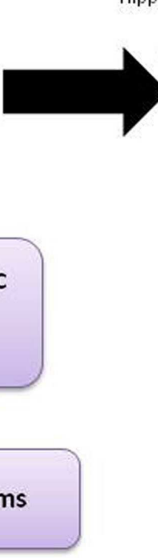

FIGURE 1 | Schematic illustration of potential effects of yoga on biomarkers and end organs based on various sources. The strength of evidence ranges from strong to preliminary for specific effects as described further in the text. Copyright Doraiswamy and Balasubramaniam, reproduced with permission in this article. 
Table 2 | Table showing checklist for RCTs according to guidelines recommended by AHRQ.

\begin{tabular}{ll}
\hline Item & Points \\
\hline Study question - clearly focused? & 1 \\
Study population & 2 \\
Randomization & 2 \\
Blinding & 2 \\
Interventions & 2 \\
Outcomes & 2 \\
Statistical analysis & 2 \\
Results & 1 \\
Discussion (including limitations and biases) & 1 \\
Funding source & 2 \\
Total & 17 \\
Score & $100 \%$ \\
\hline
\end{tabular}

impairment, studies examining cognition in the geriatric population have been included, even in the absence of formal diagnoses or specific symptoms. Studies on sub-threshold symptoms such as general well-being, stress, and coping have been excluded. Outcomes consisted of self-reported change, scores on rating scales, acceptability, and tolerance of the treatment.

The quality of RCTs was scored using the guidelines recommended by the Agency for Healthcare Research and Quality (AHRQ, 2002), with a maximum possible score of 17 . Table 2 illustrates the scoring according to the AHRQ guidelines. Study quality was additionally assessed using the Oxford Center for Evidencebased Medicine's (CEBM) Levels of Evidence, which assigns a level of evidence from 1 to 4 , where 1 indicates high quality RCTs, 2 indicates low quality RCTs, 3 suggests case-control studies, and 4 stands for case reports, case series, and low quality case-control studies (Phillips et al., 2001). Determinants of study quality have been explained in Table 3. Based on evidence levels obtained by the Oxford CEBM method, recommendation categories of A (recommended), B (suggested), or C (may be considered) have been specified for each diagnosis, as indicated by the Research and Development/University of California at Los Angeles (RAND/UCLA) Appropriateness Method (Fitch et al., 2000; Table 4). In the tables demonstrating details of individual studies for each diagnosis, the AHRQ scores, evidence level and recommendation levels have been detailed (Tables 5-9). The review has been prepared using preferred reporting items for systematic reviews and meta-analyses (PRISMA) guidelines (Moher et al., 2009).

\section{RESULTS}

Sixteen RCTs met criteria for inclusion in our review. Figure 2 illustrates the process of study extraction.

\section{YOGA FOR DEPRESSION}

Four RCTs examining the effects of yoga on depression have been included in this review. Table 5 summarizes each of these studies, including our assessments of their quality.

Shahidi et al. tested 70 elderly women (mean age of 65 years in the intervention groups and 68 years among controls) reporting subjective symptoms of depression with a baseline score of
Table 3 | Table showing levels of evidence for randomized controlled trials (based on Oxford Center for Evidence-based Medicine).

\begin{tabular}{ll}
\hline Evidence level & Study design \\
\hline 1 & High quality RCTs with narrow confidence intervals \\
2 & Low quality RCTs or high quality cohort studies \\
3 & Case-control studies \\
4 & Case series or poor case-control studies or poor \\
& cohort studies or case reports \\
\hline
\end{tabular}

High quality RCTs are those having narrow confidence intervals and $>80 \%$ followup rate.

Low quality RCTS are those with wide confidence intervals, $<80 \%$ follow-up rate. The Center for Evidence-based Medicine additionally defines low quality cohort studies as "those which have not clearly defined comparison groups and/or failed to measure exposures and outcomes in the same (preferably blinded), objective way in both exposed and non-exposed individuals and/or failed to identify or appropriately control known confounders and/or failed to carry out a sufficiently long and complete follow-up of patients."

$>10$ on the Geriatric Depression Scale (GDS) and suggested that 10 sessions of laughter yoga or exercise resulted in significant improvement of depressive symptoms from baseline and compared to a wait-list control group; however the two active treatment groups did not differ from each other (Vedamurthachar et al., 2006). The mild severity makes this study not generalizable to more severe clinical depressives.

In a 24-week study comparing the effects of yoga ( $7 \mathrm{~h}$ weekly) to Ayurveda and wait-list controls among 69 elderly individuals (mean age of 72 years), with self-report of symptoms consistent with depression and baseline mean scores on GDS corresponding to mild illness severity who were not on psychotropic medications, Krishnamurthy et al. reported that in the yoga group, there was a reduction in the scores on the GDS, from the baseline mean score of 10.6 by approximately $20 \%$ at 3 months and $40 \%$ at 6 months, a change from mild depression to no depression. This was statistically superior to the Ayurveda and wait-list control groups, neither of which demonstrated significant reduction in scores. The main limitations were the potential group interaction benefits of the yoga activity, lack of formal diagnoses according to criteria specified by the Diagnostic and Statistic Manual of Mental Disorders (DSM), relatively modest sample size, and the inclusion of only mildly depressed individuals making it difficult to generalize to more ill patients or to home based yoga (Krishnamurthy and Telles, 2007).

In a study of depression in 60 alcohol dependents males (mean age of approximately 35 years). Vedamurthachar et al. demonstrated that subjects undergoing de-addiction treatment had a statistically significant reduction in their scores on the Beck Depression Inventory (BDI), and concurrent reduction in serum cortisol levels when they received Sudarshan Kriya yoga (SKY) compared to their counterparts receiving routine inpatient care (Vedamurthachar et al., 2006). The BDI scores decreased by $75 \%$ at the end of 7 days in the yoga group. The acute alcoholism diagnosis makes it to isolate the effects of yoga versus the effects of alcohol detoxification, and further it was not clear if the subjects met stringent criteria for major depression. While this study 
Table 4 | Table showing levels of recommendation.

\begin{tabular}{|c|c|c|c|}
\hline Term & Level & Evidence levels & Explanation \\
\hline Recommended & $A$ & 1 or 2 & $\begin{array}{l}\text { Assessment supported by a substantial amount of high quality (levels } 1 \text { or } 2 \text { ) evidence } \\
\text { and/or based on consensus of clinical judgment }\end{array}$ \\
\hline Suggested & $B$ & $\begin{array}{l}1 \text { or } 2 \text { - few studies } \\
3 \text { or } 4 \text { - many studies } \\
\text { and expert consensus }\end{array}$ & $\begin{array}{l}\text { Assessment supported by sparse high grade (Level } 1 \text { or } 2 \text { ) data or a substantial amount of } \\
\text { low grade (level } 3 \text { or } 4 \text { ) data and/or clinical consensus }\end{array}$ \\
\hline May be considered & C & 3 or 4 & $\begin{array}{l}\text { Assessment is supported by low grade data without the volume to recommend more } \\
\text { highly and likely subject to revision with further studies }\end{array}$ \\
\hline
\end{tabular}

documents only a possible acute effect of yoga, it does not provide insights into longer term benefits.

A fourth study focused on treatment naive young adults (mean age of 21.5 years) with self-reported symptoms of depression and scores in the "mild mood disturbance" range on the BDI (Woolery et al., 2004). Woolery et al. found that five weekly sessions of Iyengar Yoga resulted in reduction in scores of depression, from a mean of 12.77 to a mean of 3.90, a value categorized as "normal ups and downs" at the end of 5 weeks, a statistically significant change compared to controls with a score reduction from a mean of 12.07 to 11.0 at the end of the study period. A significant reduction in anxiety and an increase in early morning cortisol level were also reported in the yoga group (Woolery et al., 2004).

None of the studies encountered adverse events in the yoga group though it was not always clear how systematically they were sought for. The drop-out rates were 0\% (Vedamurthachar et al., 2006), approximately 27\% (Krishnamurthy and Telles, 2007), and 14\% (Woolery et al., 2004). Remission and relapse prevention rates have not been determined by currently available studies. Based on our assessment of the available literature according to the RAND/UCLA Appropriateness method, Grade B evidence supporting a potential acute benefit for yoga exists in depression.

\section{YOGA FOR SCHIZOPHRENIA}

Three RCTs examining the effects of yoga on schizophrenia have been included in this review. Table 6 provides a summary. In a study based at a state psychiatric facility, comprising 18 adult patients (mean age of 37.4 in the yoga group and 48.1 among controls, but without statistical significance in age distribution) diagnosed with schizophrenia or schizoaffective disorder, Visceglia et al. compared the effects of 8 weeks of yoga as an adjunct to anti-psychotic medications with a control group receiving routine inpatient care. The authors reported a reduction in the Positive and Negative Syndrome Scale (PANSS) total score of 25.2 points, from a baseline of 85.1 in the yoga group as well as reductions of 5.9,6.0, and 13.3 in the positive syndrome, negative syndrome, and General psychopathology sub-scores, all of which were statistically superior to the controls. The secondary outcome measures of physical health and psychological health were significantly improved in the experimental group, as were informal reports of reduced aggression and improved medication compliance. The small sample size, absence of a control intervention, wide range of functionality among participants, and the short duration of follow-up are limitations of this study (Visceglia and Lewis, 2011).
Behere et al. compared the adjunctive effects of yoga with exercise wait-list controls in their 3 month study of 91 anti-psychotic stabilized adult outpatients with schizophrenia with baseline Clinical Global Impression (CGI) score less than or equal to 3. The authors reported reduction in PANSS positive and negative symptom scores by 17 and $20 \%$, respectively, statistically superior to the other two groups, as well as significant improvements in facial emotion recognition deficits, and socio-occupational functioning. Significantly higher baseline scores in the PANSS negative sub-scale and facial emotional recognition deficit in the yoga group, variation in the amount of yoga practice at home during the last 2 months of the study, limited follow-up are drawbacks of this study, and the inclusion of stable outpatients limit its generalizability to more severely ill individuals (Behere et al., 2011).

In a study of 61 anti-psychotic stabilized (mean dose of around $470 \mathrm{mg}$ /day in Chlorpromazine equivalents) inpatients and outpatients (mean age around 32 years) with schizophrenia (CGI illness severity score of 4.8 and 5.2 in the yoga and control groups) Duraiswamy et al. compared the effects of yoga with exercise, as adjuncts to anti-psychotic medications. Participants were taught yoga and exercise for 3 weeks, followed by encouragement of continued practice with monitoring of adherence. The authors reported a reduction in the total PANSS score by 25.09 points, corresponding to a moderate-to-large effect size of 0.74 in the yoga group, a greater reduction in the negative subscale (7.71 points, from a baseline of 21.9 ), but no statistically significant change between the two groups in the positive subscale. The yoga group demonstrated an improvement of sociooccupational functioning, with an effect size of 0.48 in the SocioOccupational Functioning Scale (SOFS). Notable limitations of the study include its modest sample size and unclear assessment of continued home practice of the interventions (Duraiswamy et al., 2007).

There were no adverse events, attributable to yoga reported in any of the studies, although it is not clear how this assessment had been performed. It is difficult to separate the effects of yoga from the benefits of group interaction. Assessments of change in the dose of anti-psychotics, relapse rates, and hospitalization rates have not been performed in any of the existing studies. Based on our assessment of the available literature according to the RAND/UCLA Appropriateness method, Grade B evidence supporting a potential benefit for yoga as an adjunct to anti-psychotic treatment in chronic schizophrenia. 


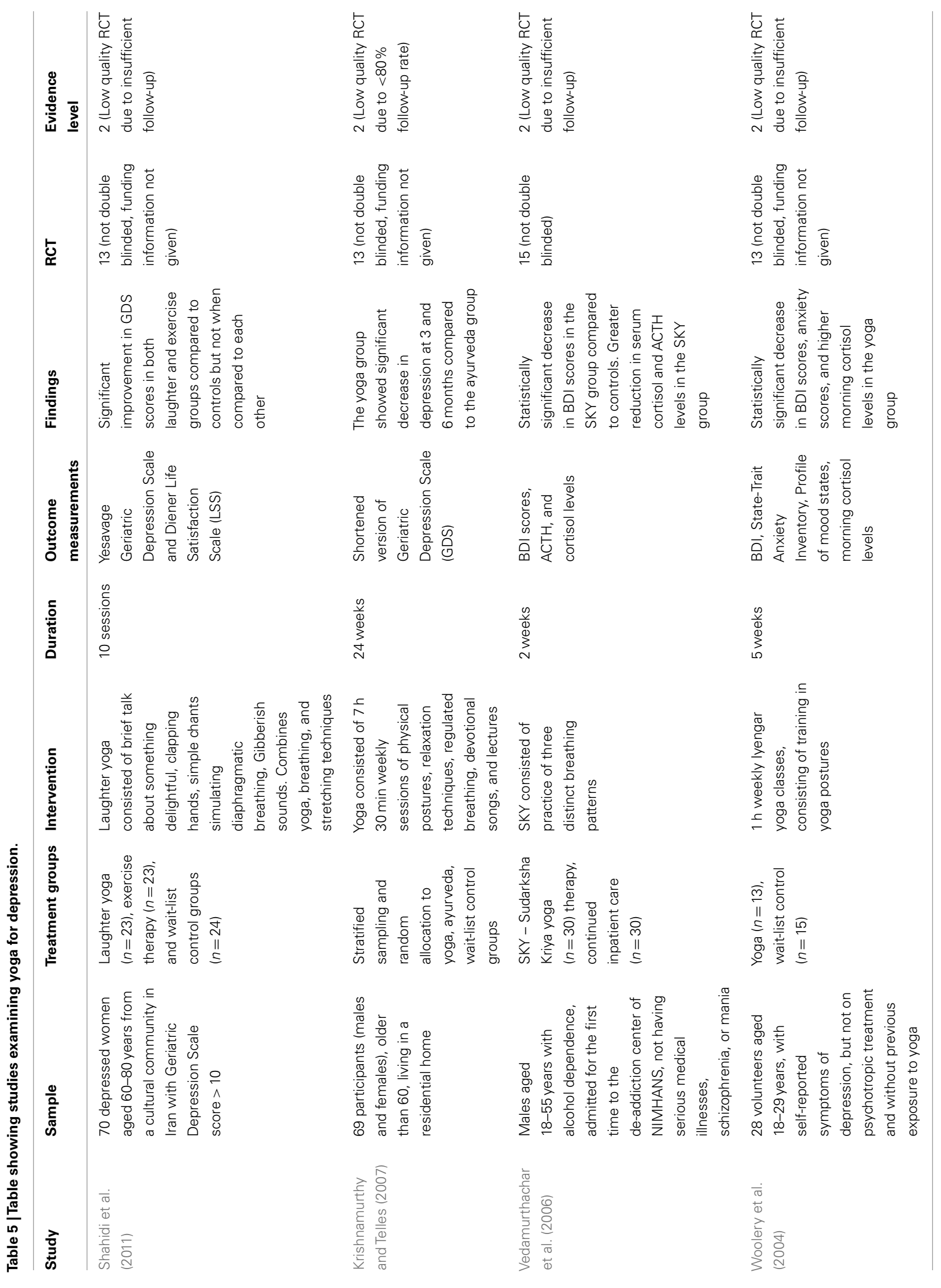




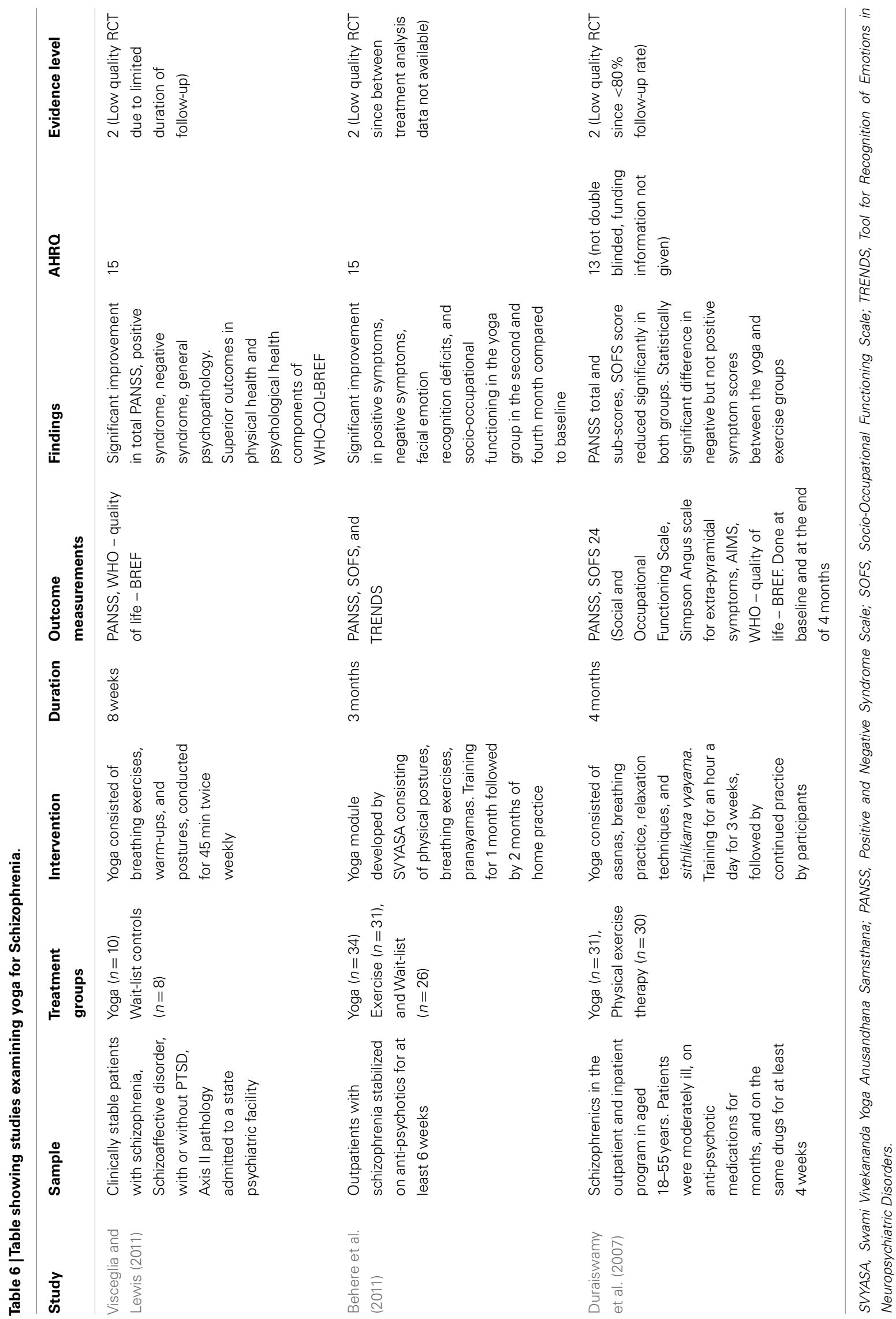




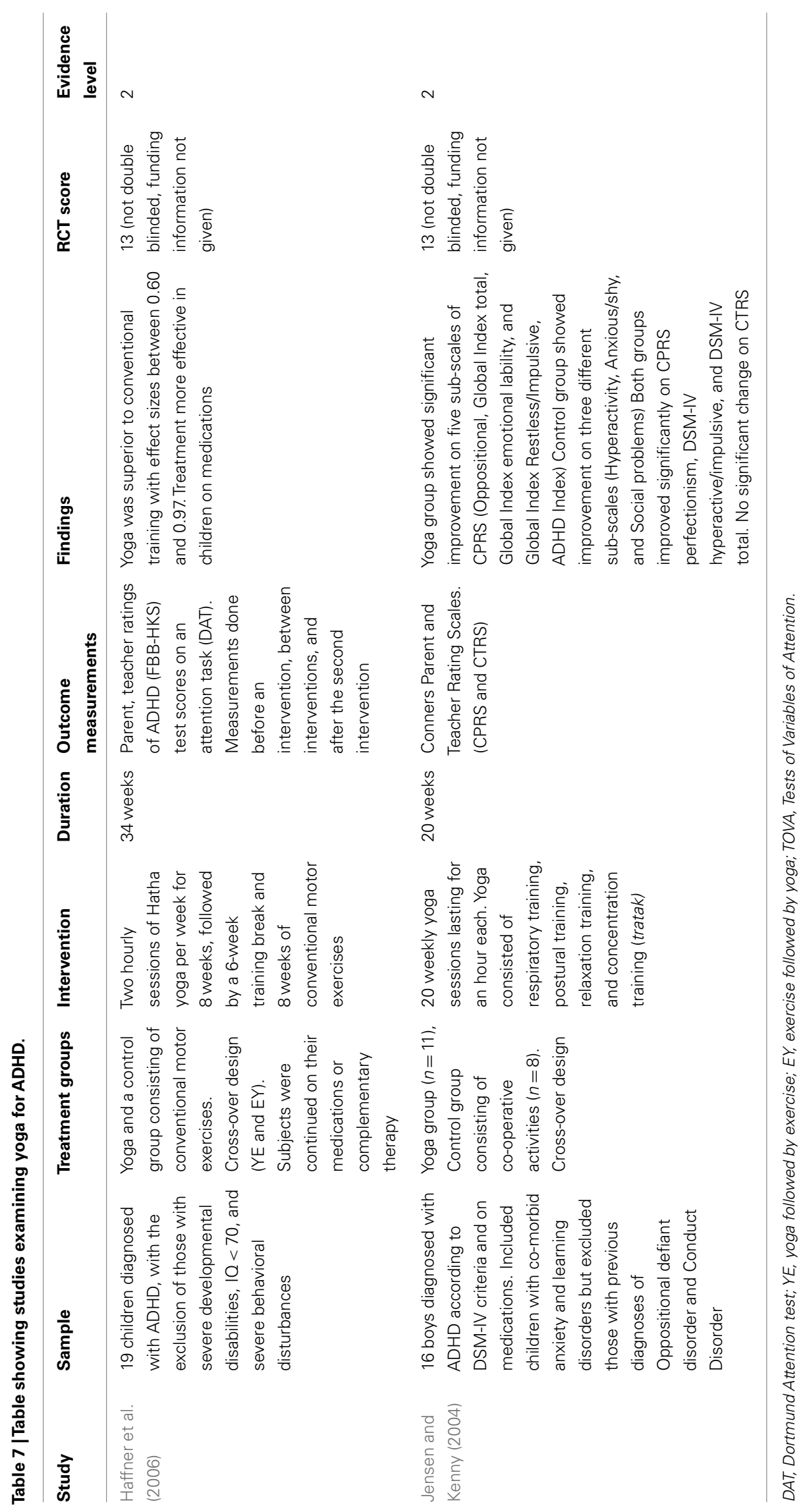




\section{YOGA FOR ATTENTION-DEFICIT HYPERACTIVITY DISORDER}

Two RCTs examining the effects of yoga on Attention-Deficit Hyperactivity Disorder (ADHD) have been included in this review (Table 7). In a cross-over study of 19 children with mean age around 10 years, diagnosed with ADHD meeting both International Classification of Diseases-10 (ICD-10) and Diagnostic and Statistical Manual of Mental Disorders-IV (DSM-IV) criteria (which included children with attention disorders, hyperkinetic disorder of social behavior, and not otherwise specified hyperkinetic disorder). Haffner et al. compared the effects of yoga with "conventional motor exercises," comprising of well known active games as adjuncts to pharmacotherapy for 34 weeks. The authors report superior efficacy of yoga with effect sizes of $0.77,0.71,0.60$, and 0.97 in the total scale, attention-deficit sub-scale, hyperactivity sub-scale, and impulsiveness sub-scale, respectively, of a German ADHD rating scale for parents and teachers. They also found a significant sequence effect on the Dartmond Attention Test (DAT), such that the group which performed yoga followed by motor exercises showed a higher improvement in scores after yoga but their mean score change at the end of the study was lower than the other group, which according to the authors may indicate that the performance gain after yoga was lost after the conventional motor exercise intervention (Haffner et al., 2006). The modest sample size, carry-over effects from the cross-over design, limited followup, and exclusion of children with severe behavioral symptoms which are frequently co-morbid with ADHD are limitations of the study.

Jensen et al. compared the effects of yoga with a control group comprising of games incorporating talking, listening, and sharing equipment for 20 weeks in their cross-over study of 16 children (mean age of 10.63 and 9.35 years in the yoga and control groups), diagnosed with ADHD according to DSM-IV criteria and continued on pharmacotherapy. They reported significant post-intervention improvement in scores on the Conners' Parent Rating Scales (CPRS), namely the Oppositional (Cohen's $d$ of 0.77), Global index Emotional lability (Cohen's $d$ of 0.79), Global Index Total (Cohen's $d$ of 0.73), Global Index Restless/Impulsive (Cohen's $d$ of 0.73), ADHD index (Cohen's $d$ of 0.29), and Perfectionism (Cohen's $d$ of 0.58 ) sub-scales but not in the Hyperactivity, anxious/shy, and social problems sub-scales, where the controls fared better. It is notable that neither group showed statistically significant improvement in scores rated by teachers, and the authors have suggested that this result may be obscured by the fact that assessments in schools occur when children are medicated, while that by parents is during unmedicated times. There were anecdotal reports by parents, of improved homework compliance and yoga being an effective calming technique during episodes or behavioral escalation. The limited follow-up limits the understanding of the maintenance effects of yoga (Jensen and Kenny, 2004).

Neither study has reported adverse events in the yoga group, although it is not clear how side effect assessment was performed. Details of pharmacotherapy for ADHD, change in dose during the course of the study have not been provided. Based on our assessment of the available literature according to the RAND/UCLA Appropriateness method, Grade B evidence supporting a potential benefit for yoga as an adjunct to pharmacotherapy in ADHD in children. 


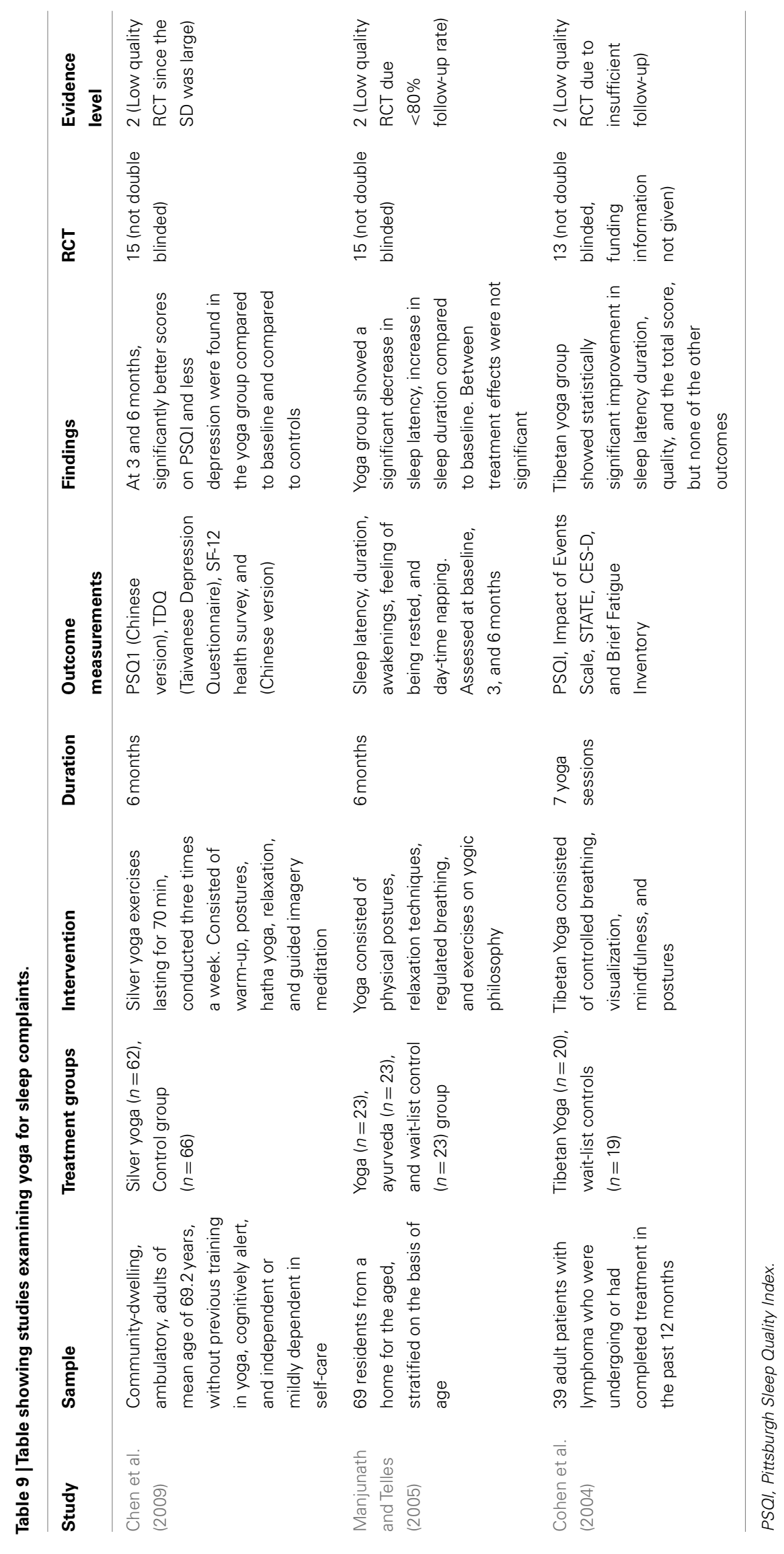




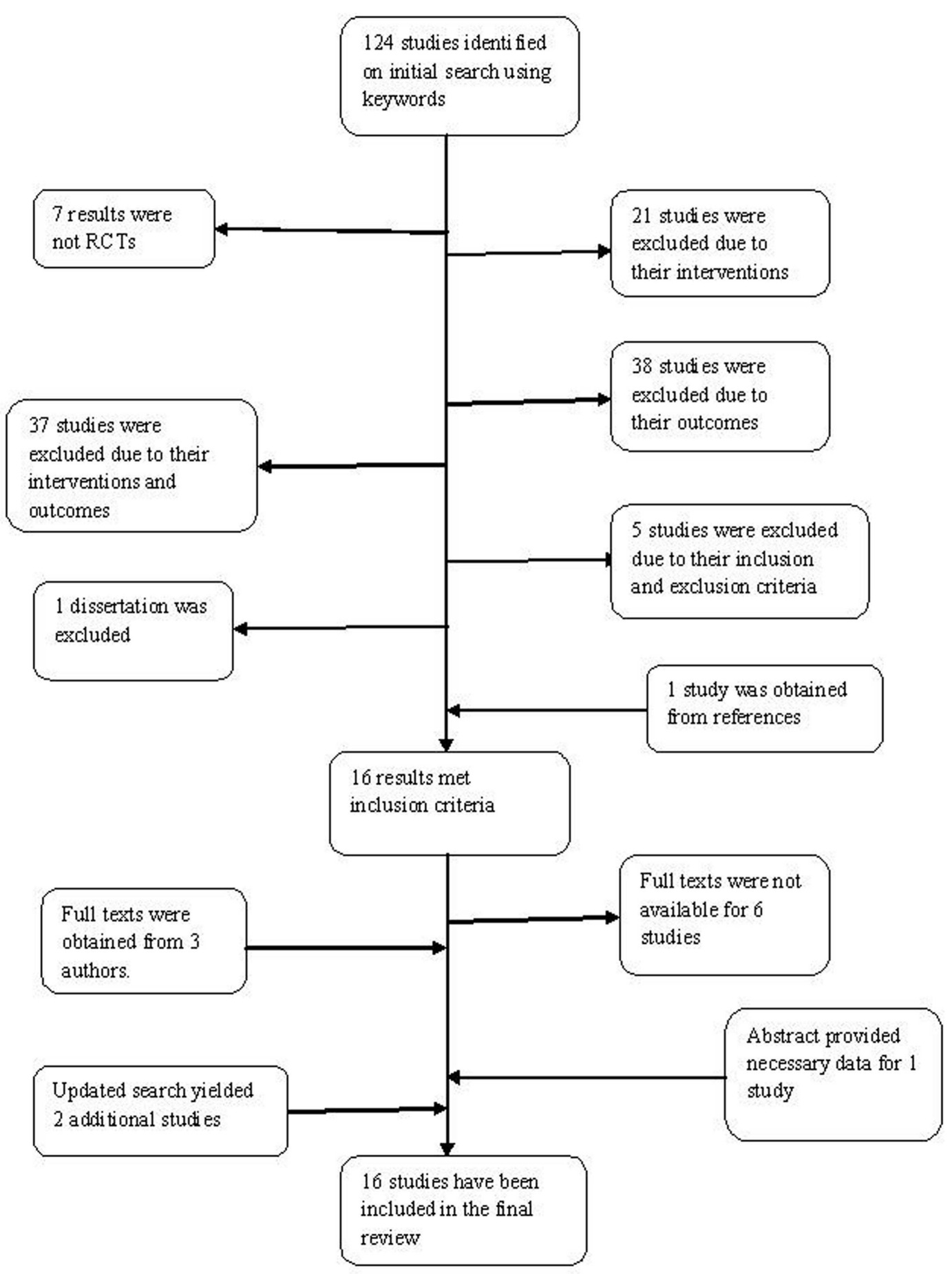

FIGURE 2 | Literature search.

\section{YOGA FOR EATING DISORDERS}

Two RCTs examining the effects of yoga on eating disorders have been included (Table 8). McIver et al. included 90 overweight or obese women (mean age of 40.1 and 42 years in the yoga and control groups) with self-reported symptoms of binge eating (listed in DSM-IV TR appendix) and a mean Binge Eating Scale (BES) score of around 28, corresponding to severe binge eating. They reported that BES score decreased by approximately $50 \%$ after 12 weeks of yoga, corresponding to an improvement from "severe" binge eating to the "absence" of binge eating, statistically superior to wait-list controls who did not demonstrate any improvement. The authors also report a lower attrition rate in the yoga group (26\%) compared 
to controls (32\%) and an increase in overall physical activity, measured by the International Physical Activity Questionnaire (IPAQ). Limitations of this study include the procurement of data by selfreport, absence of details of concurrent pharmacotherapy in the paper, and difficulty separating the true effects of yoga from that of increased contact and attention received by the yoga group during the course of the study. It would have been useful to assess whether participants perceived yoga as a way of losing weight or for overall mental health (McIver et al., 2009).

The second study in this group included 113 women (mean age of 19.56 years) reportedly "dissatisfied with their bodies," recording mean baseline scores on the Eating Disorder Diagnostic Scale (EDDS) of $26.34,30$, and 22.55 in the yoga, cognitive dissonance therapy, and wait-list control groups, respectively, where a score $>16.5$ is strongly suggestive of illness. The authors used a number of outcome measurements (see Table 9), which include assessments of eating disorder, binge eating, body shape perception, alexithymia, anxiety, and depression to compare the three groups for a duration of 6 weeks. This study reported significant improvement in the group which received therapy based on cognitive dissonance, but not in the yoga or wait-list control groups (Mitchell et al., 2007). Since only one study yielded positive results, we did not grade the evidence for this category.

\section{YOGA FOR SLEEP COMPLAINTS}

Three RCTs examining the effects of yoga on sleep complaints have been included in this review (Table 9). In their study of 139 ambulatory, community-dwelling, elderly (mean age of 69.2), cognitively able participants without previous training in yoga. Chen et al. compared the effects of yoga (three times a week for 6 months) to wait-list controls. The mean baseline total Pittsburgh Sleep Quality Index (PSQI) scores were 4.65 and 5.47, respectively, for yoga and control groups, where total score $>5$ is associated with poor sleep quality. The authors report a reduction in the total PSQI score, through 4.48 at 3 months to 3.34 at 6 months in the yoga group, statistically superior to controls who demonstrated an increase in the score, implying poor outcome. The yoga group also demonstrated statistically superior outcomes related to sleep latency, day-time dysfunction, secondary outcomes of depression, physical, and mental health perception, all of which reportedly worsened among controls. The reliance on self-report for inclusion in the study, absence of formal DSM diagnoses of either primary or secondary insomnia, baseline mean score outside of the range considered "poor sleep," absence of information about use of sleep aids limit the generalizability of these findings (Chen et al., 2009).

In a study conducted at a home for the aged in India, Manjunath et al. compared the effects of 6 months of training in yoga versus an ayurvedic preparation on 69 elderly subjects (mean age of 70.1, 72.1 , and 72.3 in the yoga, ayurveda, and wait-list control groups) with self-report of sleep impairment, but the absence of formal diagnosis of a sleep disorder at baseline. The authors reported a mean reduction in sleep latency of approximately $10 \mathrm{~min}$ and an increase in duration of approximately $60 \mathrm{~min}$ in the yoga group, a significant finding compared to the two control groups, neither of whom demonstrated comparable improvement. Of note, the sleep latency was fairly high at $25.83 \mathrm{~min}$ in the yoga group, even at the end of the study. The modest sample size, absence of formal DSM diagnoses, the presence of statistical significance within treatments but not between treatments for sleep latency are notable limitations (Manjunath and Telles, 2005).

Cohen et al. examined the effects of seven weekly sessions of Tibetan yoga (which combined training in breathing, relaxation, and postures with guided imagery), comparing it to wait-list controls on 39 adults (mean age of 51 years) with lymphoma who were either receiving chemotherapy or had received it within the past 1 year. Participants reported subjective sleep impairment and recorded baseline PSQI scores of 6.5 and 7.2, respectively, in the yoga and control groups, corresponding to "poor sleep quality" according to scoring guidelines. Formal DSM diagnoses of insomnia had not been established. The yoga group demonstrated a statistically superior reduction in the total PSQI score, a reduction from a mean of 6.5 to 5.8 , compared to controls who recorded a mean score of 8.1 at the end of the study. Scores of sleep quality (improved from 0.90 to 0.85 ), latency (improved from 1.10 to 0.75 ), and duration (improved from 0.85 to 0.89 ) were favorable in the yoga group, whereas the controls did poorly on all of the above parameters. The yoga group, but not controls showed a statistically significant reduction in the need for sleep aids - details of agents used and doses have not been specified. While there was improvement in sleep related parameters, depression, and state anxiety did not change. The modest sample size, unclear distinction between primary sleep disorders and those secondary to a mood, or anxiety disorder are drawbacks of this study (Cohen et al., 2004).

None of the studies reported adverse effects attributable to yoga, although it is not clear how they were assessed. Based on our assessment of the available literature according to the RAND/UCLA Appropriateness method, Grade C evidence supporting a potential benefit for yoga exists for sleep complaints.

\section{YOGA FOR COGNITION OR CONDITIONS INFLUENCING COGNITION}

Two studies have been included in this review, the details of which can be found on Table 10. Sharma et al. examined the adjunctive effects of 8 weeks of yoga on neurocognitive functions in 30 adults (age range between 18 and 45) meeting criteria for Major Depression, comparing it to a group which received only medications. Outcomes included measures of working memory, executive function, visual attention, task switching ability, and visual scanning, as outlined in Table 10. The authors report that while both groups demonstrate improvement in the Letter Cancelation Test (LCT), a measure of attention, concentration, and visuospatial function), Trail making tests A and B (measures of visual attention and task switching), the yoga group demonstrated superior results in LCT, and separated from the controls in the Reverse digit span test assessing short-term memory (Sharma et al., 2006). The modest sample size, lack of information about the severity of depression in the abstract are limitations and it is unclear if the improvement in these measures of cognition in the yoga group are a direct effect on cognition or secondary to greater improvement in depression mediated through yoga.

In a study of 135 elderly individuals (mean age of 71.5, 73.6, and 71.2 in the yoga, exercise, and wait-list groups, respectively). Oken et al. compared the effects of yoga with exercise and waitlist controls over 6 months, focusing on measures of alertness 


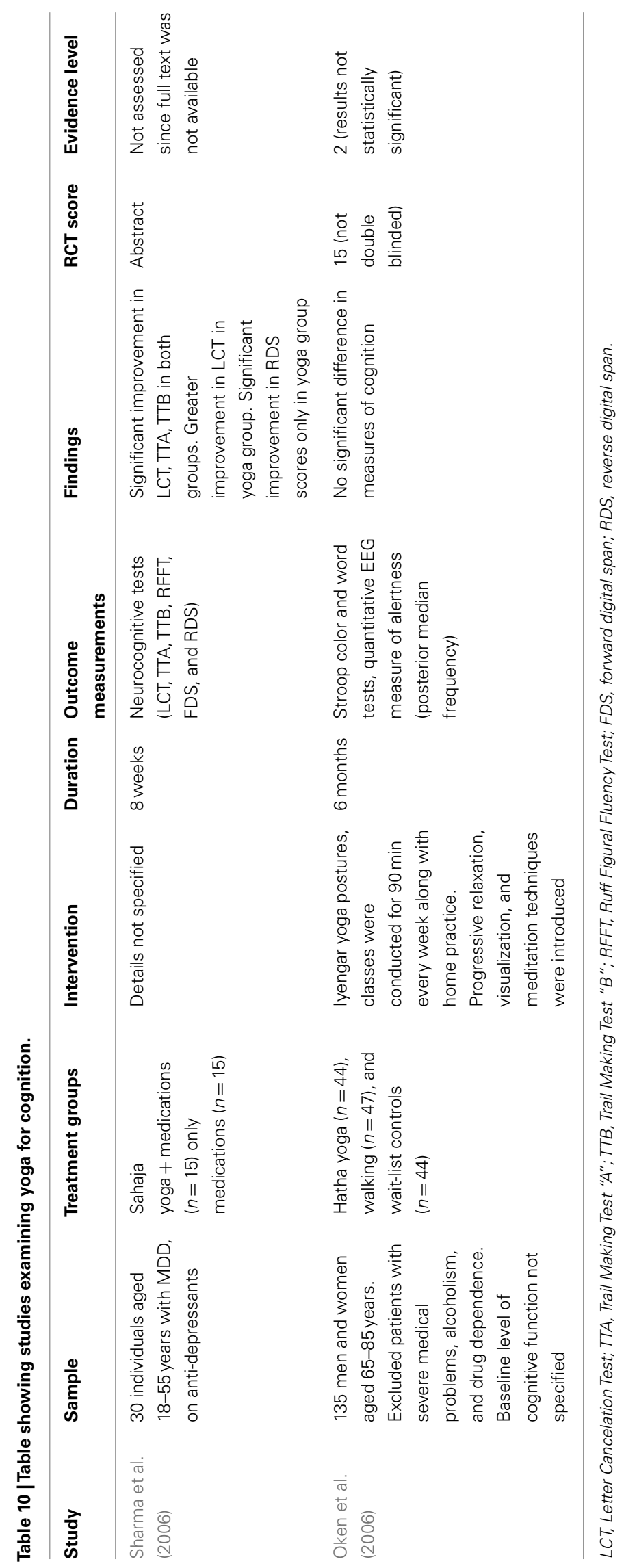


using EEG and the Stroop Color and word tests. Baseline cognitive assessments are not reported to have been performed; individuals with severe medical illnesses were excluded, as were those with experience in yoga over the last 6 months. Significant changes in measures of cognition were not demonstrated in any of the groups. The yoga group did demonstrate improvement in quality of life measures related to a sense of well-being as physical measures such as forward flexibility and timed one leg standing. The negative results notwithstanding, the absence of specification of baseline cognitive status is a drawback of this study (Oken et al., 2006).

\section{DISCUSSION \\ KEY FINDINGS}

To our knowledge, this is the first review to systematically examine the published literature on benefits of yoga for several major psychiatric illnesses. Based on our assessment of the available literature according to the RAND/UCLA Appropriateness method, Grade B evidence supporting a potential acute benefit for yoga exists in depression (four RCTs), as an adjunct to medications in Schizophrenia (three RCTs) and ADHD (two RCTs), and Grade $\mathrm{C}$ evidence supports the benefit of yoga for sleep complaints (three RCTs).

Studies have found reasonable benefit in mild depression, even in the absence of pharmacotherapy. Studies of yoga in schizophrenia have yielded evidence of benefit as an adjunct to medications in improving positive and negative symptoms, quality of life, and socio-occupational functioning. The RCTs examining yoga in ADHD have demonstrated moderate-large effect sizes, comparable according to the authors, to other alternative therapies such as biofeedback and relaxation in ADHD (Haffner et al., 2006). Three RCTs suggest substantial benefit for sleep complaints, although the absence of formal DSM diagnoses in these studies is limiting. RCTs in cognitive disorders and eating disorders yielded conflicting results. Of note, Grade B implies that the assessment is supported by sparse high grade data or a substantial amount of low grade data and/or clinical consensus and Grade C suggests that the assessment is supported by low grade data without the volume to recommend more highly and likely subject to revision with further studies (Fitch et al., 2000).

\section{LIMITATIONS BASED ON SEARCH STRATEGY AND INCLUSION/EXCLUSION CRITERIA}

Although yoga has been used as a treatment for a wide variety of psychiatric conditions and distress, we have focused on the major broad categories of psychiatric disorders, namely depression, schizophrenia, eating disorders, ADHD, sleep complaints, and cognitive impairments. We excluded studies on sub-threshold symptoms such as coping, general well-being as well as studies conducted on individuals without psychiatric diagnosis. This was

\section{REFERENCES}

Agency for Healthcare Research and Quality. (2002). Systems to Rate the Strength of Scientific Evidence. Evidence Report/Technological Assessment No. 47. Available at: http://archive.ahrq.gov/clinic/ epcsums/strengthsum.pdf

Barrows, K. A., and Jacobs, B. P. (2002). Mind-body medicine: an introduction and review of the literature. Med. Clin. North Am. 86, 11-31.

done to minimize the possibility that observed effects are merely a reactive change to a new event in normal individuals. Our search term "anxiety" yielded studies on post-traumatic stress, state, and trait anxiety but these studies had specifically excluded individuals with pre-established psychiatric diagnoses and hence did not make it to the final review.

\section{LIMITATIONS BASED ON STUDY METHODOLOGY}

Few studies have provided details on how randomization had been performed. Studies included in our review consist of various subtypes of yoga and the description of the intensity of yoga has not been specified in many studies. The number of studies for each sub-type of yoga is very small, therefore, for the purpose of our review, which is the first of its kind, we considered subtypes which included similar basic components, namely controlled breathing, relaxation, and postural training to be equivalent. Due to the nature of the intervention, blinding of subjects is challenging, while information regarding blinding of the assessor has not been provided in most studies. Analogous to other interventions such as exercise, where they may be effects of group intervention, it is difficult to isolate benefits of being in a group from that derived from yoga alone in our studies. This may be particularly the case in studies with wait-list controls, where it is difficult to establish if the observed changes are due to the effect of yoga or merely expectation. The sample sizes are small in many studies and the generalizability of benefits noted in participants who demonstrate the motivation to participate and comply in studies of yoga may be questionable. The severity of illness has varied across studies, and it is of concern if the findings from results of mildly ill individuals (such as the depression studies) can be extrapolated to those with severe illness. Although adverse effects have not been reported in these studies, details of how the assessment had been done are lacking.

\section{CONCLUSION}

Our systematic review finds emerging scientific evidence to support a role for yoga in treating depression, sleep complaints consistent with both popular beliefs and biological studies, and having adjunctive value in schizophrenia and ADHD. The evidence in other disorders remains less well established. Given the growing popularity of yoga, it would be important for the field to attempt to replicate and extend these findings in larger, multicenter, randomized, blinded (at least single blinded) studies with the control group receiving alternative treatments, preferably using Good clinical practice (GCP) guidelines. Biomarker research, such as through functional magnetic resonance imaging (MRI) and Positron Emission Tomography (PET) studies, and molecular markers (genomics, metabolomics, and proteomics), would facilitate greater scientific understanding at a neurobiological level, of this 5000-year-old revered practice.

Behere, R. V., Arasappa, R., Jagannathan, A., Varambally, S., Venkatasubramanian, G., Thirthalli, J., et al. (2011). Effects of yoga therapy on facial emotion recognition deficits, symptoms and functioning in patients with schizophrenia. Acta Psychiatr. Scand. 123, 147-153.

Carei, T. R., Fyfe-Johnson, A. L., Breuner, C. C., and Brown, M. A. (2010). Randomized controlled clinical trial of yoga in the treatment of eating 
disorders. J. Adolesc. Health 46, 346-351.

Chen, K. M., Chen, M. H., Chao, H. C., Hung, H. M., Lin, H. S., and Li, C. H. (2009). Sleep quality, depression state, and health status of older adults after silver yoga exercises: cluster randomized trial. Int. J. Nurs. Stud. 46, 154-163.

Cohen, L., Warneke, C., Fouladi, R. T., Rodriguez, M. A., and Chaoul-Reich, A. (2004). Psychological adjustment and sleep quality in a randomized trial of the effects of a Tibetan yoga intervention in patients with lymphoma. Cancer 100, 2253-2260.

Cook, J. (n.d.). Not all yoga is created equal. Yoga J. Available at: http: //www.yogajournal.com/basics/165 (accessed January 13, 2012).

Duraiswamy, G., Thirthalli, J., Nagendra, H., and Gangadhar, B. N. (2007). Yoga therapy as an addon treatment in the management of patients with schizophrenia - a randomized controlled trial. Acta Psychiatr. Scand. 116, 226-232.

Fitch, K., Bernstein, S. J., Aguilar, M. S., Burnand, B., La Calle, J. R., Lazaro, P., et al. (2000). The RAND/UCLA Appropriateness Method User's Manual. Available at: http://www.rand. org

Goeree, R., Farahati, F., Burke, N., Blackhouse, G., O'Reilly, D., Pyne, J., et al. (2005). The economic burden of schizophrenia in Canada in 2004. Curr. Med. Res. Opin. 21, 2017-2028.

Goldberg, D., Privett, M., Ustun, B., Simon, G., and Linden, M. (1998). The effects of detection and treatment on the outcome of major depression in primary care: a naturalistic study in 15 cities. Br. J. Gen. Pract. 48, 1840-1844.

Haffner, J., Roos, J., Goldstein, N., Parzer, P., and Resch, F. (2006). Zur Wirksamkeit korperorientierter Therapieverfahren bei der Behandlung hyperaktiver Storungen: Ergebnisse einer [The Effectiveness of Body-Oriented Methods of Therapy in the Treatment of Attention-Deficit Hyperactivity Disorder (ADHD): Results of a Controlled Pilot Study]. Z Kinder Jugendpsychiatr Psychother. 34, 37-47.

Janakiramaiah, N., Gangadhar, B. N., Nagavenkatesha-Murthy, P. J., Harish, M. G., Subbakrishna, D. K., and Vedamurthachar, A. (2000). Antidepressant efficacy of sudarshan kriya yoga (SKY) in melancholia: a randomized comparison with electroconvulsive therapy and imipramine. J. Affect. Disord. 57, 255-259.
Janakiramaiah, N., Gangadhar, B. N., Nagavenkatesha-Murthy, P. J., Shetty, T. K., Subbakrishna, D. K., Meti, B. L., et al. (1998). Therapeutic efficacy of sudarshan kriya yoga (SKY) in dysthymic disorder. NIMHANS J. 17, 21-28.

Javnbakht, M., Hejazi Kenari, R., and Ghasemi, M. (2009). Effects of yoga on depression and anxiety of women. Complement. Ther. Clin. Pract. 15, 102-104.

Jensen, P. S., and Kenny, D. T. (2004). The effects of yoga on the attention and behavior of boys with attention-deficit/hyperactivity disorder (ADHD). J. Atten. Disord. 7, 205-216.

Kamei, T., Toriumi, Y., Kimura, H., Ohno, S., Kumano, H., and Kimura, K. (2000). Decrease in serum cortisol during yoga exercise is correlated with alpha wave activation. Percept. Mot. Skills 90, 1027-1032.

Katzman, M. A., Vermani, M., Gerbarg, P. L., Brown, R. P., Iorio, C., Davis, M., et al. (2012). A multicomponent yoga-based, breath intervention program as an adjunctive treatment in patients suffering from Generalized Anxiety Disorder with or without comorbidities. Int. J. Yoga 5, 57-65.

Kessler, R. C., Aguilar-Gaxiola, S., Alonso, J., Chatterji, S., Lee, S., Ormel, J., et al. (2009). The global burden of mental disorders: an update from the WHO World Mental Health (WMH) surveys. Epidemiol. Psichiatr. Soc. 18, 23-33.

Kessler, R. C., Chiu, W. T., Demler, O., Merikangas, K. R., and Walters, E. E. (2008). Prevalence, severity, and co-morbidity of 12 month DSMIV disorders in the National Comorbidity Survey Replication. Arch. Gen. Psychiatry 62, 617-627.

Krishnamurthy, M. N., and Telles, S. (2007). Assessing depression following two ancient Indian interventions: effects of yoga and ayurveda on older adults in a residential home. J. Gerontol. Nurs. 33, 17-23.

Libby, D. J., Reddy, F., Pilver, C. E., and Desai, R. A. (2012). The use of yoga in specialized VA PTSD treatment programs. Int. J. Yoga Therap. 22, 79-88.

Lieberman, J. A., Stroup, S., McEvoy, J. P., Swartz, M. S., Rosenheck, R. A., Perkins, D. O., et al. (2005). Clinical Antipsychotic Trials of Intervention Effectiveness (CATIE) investigators. Effectiveness of antipsychotic drugs in patients with chronic schizophrenia. N. Engl. J. Med. 353, 1209-1223.

Manjunath, N. K., and Telles, S. (2004). Spatial and verbal memory test scores following yoga and fine arts camps for school children. Indian J. Physiol. Pharmacol. 48, 353-356.

Manjunath, N. K., and Telles, S. (2005). Influence of yoga and ayurveda on self-rated sleep in a geriatric population. Indian J. Med. Res. 121, 683-690.

Maussavi, S., Chatterji, S., Verdes, E., Tandon, A., Patel, V., and Ustun, B. (2007). Depression, chronic diseases and decrements in health: results from the World Health Surveys. Lancet 370, 851-858.

McIver, S., O'Halloran, P., and McGartland, M. (2009). Yoga as a treatment for binge eating disorder: a preliminary study. Complement. Ther. Med. 17, 196-202.

Mitchell, K. S., Mazzeo, S. E., Rausch, S. M., and Cooke, K. L. (2007). Innovative interventions for disordered eating: evaluating dissonance based and yoga interventions. Int. J. Eat. Disord. 40, 120-128.

Moher, D., Liberati, A., Tetzlaff, J., Altman. D. G, and The PRISMA Group. (2009). Preferred reporting items for systematic reviews and meta-analyses. The PRISMA Statement. PLoS Med. 6:e1000097. doi:10.1371/journal.pmed.1000097

Moul, D. E., Nofzinger, E. A., and Pilkonis, P. A. (2002). Symptom reports in severe chronic insomnia. Sleep 25 553-563.

National Sleep Foundation. (2005). 2005 Sleep in America Poll. Washington, DC: National Sleep Foundation.

Oken, B. S., Zajdel, D., Kishiyama, S. Flegal, K., Dehen, C., Haas, M., et al. (2006). Randomized, controlled, six-month trial of yoga in healthy seniors: effects on cognition and quality of life. Altern. Ther. Health Med. 12, 40-47.

Phillips, B., Ball, C., Sackett, D., Badenoch, D., Straus, S., Haynes, B., et al. (2001). Oxford Centre for EvidenceBased Medicine - Levels of Evidence. Available at: http://www.cebm.net/ index.aspx?0=1025 [March 2009]

Phillips, W. T., Kiernan, M., and King, A. C. (2003). Physical activity as a nonpharmacological treatment for depression: a review. Complement. Health Pract. Rev. 8 139-152.

Shahidi, M., Mojtahed, A., Modabbernia, A., Mojtahed, M., Shafiabady, A., Delavar, A., et al. (2011). Laughter yoga versus group exercise program in elderly depressed women: a randomized controlled trial. Int. J. Geriatr. Psychiatry 26, 322-327.

Shannahoff-Khalsa, D. S., Ray, L. E., Levine, S., Gallen, C. C., Schwartz,
B. J., and Sidorowich, J. J. (1999). Randomized controlled trial of yogic meditation techniques for patients with obsessive-compulsive disorder. CNS Spectr. 4, 34-47.

Sharma, V. K., Das, S., Mondal, S. Goswami, U., and Gandhi, A. (2006). Effect of sahaj yoga on neurocognitive functions in patients suffering from major depression. Indian J. Physiol. Pharmacol. 50, 375-383.

Streeter, C. C., Jensen, J. E., and Perlmutter, R. M. (2007). Yoga asana sessions increase brain GABA levels: a pilot study. J. Altern. Complement. Med. 13, 419-426.

Telles, S. (2010). A theory of disease from ancient yoga texts. Med. Sci. Monit. 16, LE9.

Trivedi, M. H., Rush, A. J., and Wisniewski, S. R. (2006). Evaluation of outcomes with citalopram for depression using measurementbased care in STAR $* \mathrm{D}$ : implications for clinical practice. Am. J. Psychiatry $163,28-40$

Uebelacker, L. A., Tremont, G., EpsteinLubow, G., Gaudiano, B. A., Gillette, T., Kalibatseva, Z., et al. (2010). Open trial of Vinyasa Yoga for persistently depressed individuals: evidence of feasibility and acceptability. Behav. Modif. 34, 247.

Vedamurthachar, A., Janakiramaiah, N., Hegde, J. M., Shetty, T. K., Subbakrishna, D. K., Sureshbabu, S. V., et al. (2006). Antidepressant efficacy and hormonal effects of sudarshana kriya yoga (SKY) in alcohol dependent individuals. J. Affect. Disord. 94 249-253.

Vempati, R. P., and Telles, S. (2000). Baseline occupational stress levels and physiological responses to a two day stress management program. $J$. Indian Psychol. 18, 33-37.

Vialatte, F. B., Bakardjian, H., Prasad, R., and Cichocki, A. (2008). EEG paroxysmal gamma waves during Bhramari Pranayama: a yoga breathing technique. Conscious. Cogn. 18, 977-988.

Visceglia, E., and Lewis, S. (2011). Yoga therapy as an adjunctive treatment for schizophrenia: a randomized, controlled pilot study. J. Altern. Complement. Med. 17, 601-607.

Woolery, A., Myers, H., Sternlieb, B., and Zeltzer, L. (2004). A yoga intervention for young adults with elevated symptoms of depression. Altern. Ther. Health Med. 10, 60-63.

World Health Organization. (2008). The Global Burden of Disease: 2004 Update. Geneva: WHO Press.

World Health Organization. (2011). Global Status Report on 
Non-Communicable Diseases 2010. Geneva: WHO.

Xiong, G. L., and Doraiswamy, P. M. (2009). Does meditation enhance cognition and brain plasticity? Ann. N. Y. Acad. Sci. 1172, 63-69.

Conflict of Interest Statement: P. Murali Doraiswamy has received research grants and/or advisory fees/honoraria from several government agencies, media, and pharmaceutical companies. He owns stock in Sonexa and Clarimedix.

Received: 09 September 2012; paper pending published: 12 October 2012; accepted: 20 December 2012; published online: 25 January 2013.
Citation: Balasubramaniam M, Telles $S$ and Doraiswamy PM (2013) Yoga on our minds: a systematic review of yoga for neuropsychiatric disorders. Front. Psychiatry 3:117. doi: 10.3389/fpsyt.2012.00117

This article was submitted to Frontiers in Affective Disorders and Psychosomatic Research, a specialty of Frontiers in Psychiatry.
Copyright (c) 2013 Balasubramaniam, Telles and Doraiswamy. This is an openaccess article distributed under the terms of the Creative Commons Attribution License, which permits use, distribution and reproduction in other forums, provided the original authors and source are credited and subject to any copyright notices concerning any third-party graphics etc. 